Pesq. Vet. Bras. 35(3):258-264, março 2015

DOI: $10.1590 / \mathrm{S} 0100-736 \mathrm{X} 2015000300008$

\title{
Tipagem molecular e resistência aos antimicrobianos em isolados de Escherichia coli de frangos de corte e de tratadores na Região Metropolitana de Curitiba, Paraná ${ }^{1}$
}

\author{
Arnildo Korb ${ }^{2 *}$, Eleusis R. de Nazareno ${ }^{3}$, Libera D. Costa ${ }^{4}$, Keite da Silva Nogueira ${ }^{4}$, \\ Paulo R. Dalsenter ${ }^{5}$, Felipe F.B. Tuon ${ }^{4}$ e Maria C. Pomba ${ }^{6}$
}

\begin{abstract}
Korb A., Nazareno E.R., Costa L.D., Nogueira K.S., Dalsenter P.R., Tuon F.F.B. \& Pomba M.C. 2015. [Molecular typing and antimicrobial resistance in isolates of Escherichia coli from poultry and farmers in the Metropolitan Region of Curitiba, Paraná.] Tipagem molecular e resistência aos antimicrobianos em isolados de Escherichia coli de frangos de corte e de tratadores na Região Metropolitana de Curitiba, Paraná. Pesquisa Veterinária Brasileira 35(3):258-264. Departamento de Enfermagem, Universidade do Estado de Santa Catarina, Rua Sete de Setembro 91D, Chapecó, SC 89801-140, Brazil. E-mail: arnildo.korb@udesc.br

This study examined the profile of antimicrobial resistance among isolates of Escherichia coli from poultry intensive farming and free-range systems and their farmers. For technique of Gel Electrophoresis Pulsed Field (PFGE) examined the similarity between isolates from poultry intensive farming and their farmers. From 60 samples of poultry feces from intensive farming systems, 60 of free-range extensive systems and 20 of farmers of each segment, the E. coli was isolated and submitted to the test of susceptability to 12 antimicrobials. 24 isolates of $E$. coli of poultry from intensive farming systems and eight $E$. coli isolates from farmers poultry intensive farming were analyzed via technique of PFGE. In intensive farming systems poultry, $100 \%$ resistence to ampicillin was verified, $43 \%$ to cefotaxime, $48 \%$ to ceftriaxone, $62 \%$ to nalidixic acid, $23 \%$ to enrofloxacin, $23 \%$ to ciprofloxacin, $83 \%$ to tetracycline and $45 \%$ to trimetroprim-sulfametoxazol. In the strains of free-range extensive systems, resistance was $20 \%, 0 \%, 0 \%, 5 \%, 2 \%, 4 \%, 33 \%$ and $8 \%$, respectively. Resistance to fosfomycin and to nitrofuratoin was found in isolates of poultry from free-range extensive systems. In farmers from intensive farming systems, the resistance to ampicillin was $60 \%, 25 \%$ to ciprofloxacin and $45 \%$ to tetracycline, whereas in farmers from free-range extensive systems, it was $20 \%, 5 \%$ and $30 \%$, respectively. In the isolates of $E$. coli poultry from free-range extensive systems, $46.6 \%(28 / 60)$ presented themselves as susceptible to all tested antimicrobials in comparison to intensive farming systems in which $81,6 \%(49 / 60)$ were multiresistant. Seven clusters of isolates from poultry showed similarity above $80 \%$. Out of these, two clusters of isolates of poultry from different aviaries presented superior clonality to $95 \%$. Furthermore three clusters isolates of poultry and farmers showed similarity greater than $80 \%$, but only one cluster isolate of attendant and poultry were from the same aviary.
\end{abstract}

INDEX TERMS: Intensive livestock farming and free-range poultry, Escherichia coli, antimicrobial, molecular typing.

\footnotetext{
${ }^{1}$ Recebido em 24 de julho de 2014.

Aceito para publicação em 23 de março de 2015.

${ }^{2}$ Departamento de Enfermagem, Universidade do Estado de Santa Catarina (UDESC), Rua Sete de Setembro 91D, Centro, Chapecó, SC 89801-140, Brasil. Pesquisa de doutorado com apoio CAPES. *Autor para correspondência: arnildo.korb@udesc.br

${ }^{3}$ Departamento de Saúde Comunitária, Universidade Federal do Paraná (UFPR), Rua Padre Camargo 280, 3ํandar, Curitiba, PR 80060-240, Brasil.
}

\footnotetext{
${ }^{4}$ Hospital de Clinicas, UFPR, Rua Gen. Carneiro 181, Alto da Glória, Curitiba, PR 80060-900.

${ }^{5}$ Departamento de Farmacologia, UFPR, Rua Cel. Francisco H. dos Santos 100, Centro Politécnico, Jardim das Américas, Cx. Postal 19031, Curitiba, PR 81531-980.

${ }^{6}$ Hospital Veterinário, Universidade de Lisboa (ULisboa), Avenida da Universidade Técnica, Polo Universitário da Ajuda, Lisboa, 1300-477, Portugal.
} 
RESUMO.- Este estudo verificou o perfil de resistência aos antimicrobianos entre isolados de Escherichia coli de frangos de corte de criação intensiva e de subsistência e dos respectivos tratadores e a similaridade genotípica entre isolados de E.coli de frangos de corte de criação intensiva e isolados de E. coli de tratadores de frangos de criação intensiva pela técnica de Eletroforese em Gel de Campo Pulsado (PFGE). 60 amostras de fezes de frangos de criação intensiva, 60 de frangos de corte de criação de subsistência (caipira) e 20 amostras dos tratadores de frangos de criação intensiva e 20 de tratadores de frangos de criação de subsistência. E. coli foram isoladas, identificadas e submetidas ao teste de suscetibilidade a 12 antimicrobianos. Pela PFGE foram analisados 24 isolados de E. coli de frangos de corte de criação intensiva e oito de tratadores. Em isolados E. coli de frangos de criação intensiva a resistência para a ampicilina foi de $100 \%$, cefotaxima $43 \%$, ceftriaxona $48 \%$, ácido nalidíxico $62 \%$, enrofloxacina $23 \%$, ciprofloxacina $23 \%$, tetraciclina $83 \%$ e $45 \%$ para trimetoprim-sulfametoxazol. Nos isolados de frangos de criação de subsistência foi de $20 \%, 0 \%, 0 \%, 5 \%, 2 \%, 4 \%, 33 \%$ e $8 \%$, respectivamente. Resistência à fosfomicina e à nitrofurantoína foi encontrada em isolados de frangos de criação de subsistência. Em isolados de E. coli de tratadores de frangos de corte de criação intensiva a resistência para ampicilina foi de $60 \%$, para ciprofloxacina $25 \%$ e para tetraciclina $45 \%$, enquanto nos tratadores de subsistência foram de $20 \%$, 5\% e $30 \%$, respectivamente. Isolados de E. coli de frangos em criação de subsistência apresentaram 46,6\%(28/60) de suscetibilidade a todos os antimicrobianos testados enquanto que na criação intensiva 81\%(49/60) foram multirresistentes. Sete clusters de isolados de E. coli de frangos de diferentes aviários apresentaram similaridade acima de $80 \%$, e dois destes foram superiores a $95 \%$. Três clusters de isolados de frangos e de tratadores apresentaram similaridade superior a $80 \%$. Somente um destes clusters foi de isolado de tratador e de frango do mesmo aviário.

TERMOS DE INDEXAÇÃO: Frangos de corte de criação intensiva e de subsistência, Escherichia coli, antimicrobianos, tipagem molecular.

\section{INTRODUÇÃO}

Na avicultura de criação intensiva Escherichia coli patogênica aviária (APEC) causa infecções respiratórias, entéricas, pericardite, perihepatite, septicemia e a dermatite necrótica (Celulite) (Dziva \& Stevens 2008). A condenação de carcaças nos abatedouros por conta da celulite provoca elevados prejuízos aos produtores, assim como gastos expressivos na utilização de antimicrobianos para controlá-la (Blanco et al. 1997). Existem evidências de que as resistências aos antimicrobianos em bactérias da microbiota de frangos de corte de criação intensiva possam ser transferidas para o ser humano por ingestão de carnes cruas ou material fecal-oral (Johnson et al 2007).

0 "Strategic and Technical Advisory Group" (STAG- AMR) da Organização Mundial da Saúde (WHO 2013) na sua última reunião de setembro de 2013, recomenda o uso prudente dos antimicrobianos na saúde humana e na produção animal. Esta recomendação procede em virtude de registros de mortes humanas por infecções causadas por bactérias multirresistentes e da dificuldade de introdução de novos antibióticos no mercado. A WHO propõe a preservação de categorias de antimicrobianos criticamente importantes para a saúde humana, principalmente os de utilização na produção animal como as cefalosporinas de terceira e quarta geração e as fluorquinolonas (AGISAR 2012). 0 uso frequente de antimicrobianos na avicultura seleciona resistências em bactérias comensais do sistema digestório, em particular em E. coli, as quais podem por contato direto serem transferidas para tratadores por colonização do trato digestório (Van den Bogaard et al. 2001, Ewers et al. 2012, Knöbl et al. 2012, Manges \& Johnson 2012). Mundialmente, esta bactéria prevalece em infecções de animais e seres humanos, como no trato urinário, resultando em altos custos aos sistemas de saúde (Gould 2010, Dheilly 2011). No tratamento de infecções urinárias humanas a ciprofloxacina, metabólito da enrofloxacina (Shah 1991) está entre as mais prescritas (Sheerin 2011).

Este estudo objetivou verificar o perfil de resistência aos antimicrobianos em isolados de E. coli de frangos de corte de criação intensiva, frangos de criação de subsistência e dos respectivos tratadores. Além de verificar a similaridade genotípica entre isolados de $E$. coli de frangos de corte de criação intensiva com os isolados de E. coli de tratadores de frangos de criação intensiva.

\section{MATERIAL E MÉTODOS}

Amostras. A colheita de fezes de frangos ocorreu em 20 aviários de criação intensiva e em 20 propriedades de criação de subsistência, localizadas na região metropolitana de Curitiba. A colheita das amostras foi realizada em frangos de corte com idade mínima de 25 dias. De julho a setembro de 2012, de cada aviário foram coletadas três amostras de fezes frescas por aviário, totalizando 120 amostras. 60 de criação intensiva (CI) e 60 de criação de subsistência (CS).

Na criação intensiva, em município pertencente à região metropolitana de Curitiba, foram colhidas amostras de fezes de tratadores (TI) do sexo masculino $(n=16)$ e do sexo feminino $(n=4)$. Todos os tratadores (20) da criação de subsistência (TS) foram do sexo feminino.

Isolamento e identificação de $\boldsymbol{E}$. coli. No controle do teste de suscetibilidade foi utilizada a cepa E. coli ATCC 35218. As amostras foram semeadas em ágar em MacConkey ágar (BD Difco) e incubadas a $37^{\circ} \mathrm{C}$, em aerobiose durante 18 a 24 horas. Uma colônia com características morfológicas de $E$. coli foi transferida de cada placa para meio ágar sangue (Oxoid) e incubada nas condições anteriores. A identificação da espécie foi realizada com os testes bioquímicos em Citrato de Simmnons, TSI, SIM, VM e VP (Dufour-Zavala 2008). Os isolados de E. coli com características positivas foram identificados fenotipicamente como E. coli.

Teste de suscetibilidade. 0 teste de suscetibilidade aos antimicrobianos foi realizado pela técnica de disco-difusão (Bauer et al. 1966) usando o meio de Muller-Hinton (Oxoid) e os discos

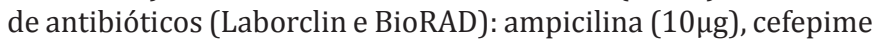
$(30 \mu \mathrm{g})$, cefotaxima $(30 \mu \mathrm{g})$, ceftriaxona $(30 \mu \mathrm{g})$, ceftazidima(30 $\mu \mathrm{g})$, ácido nalidíxico $(10 \mu \mathrm{g})$, enrofloxacina $(5 \mu \mathrm{g})$, ciprofloxacina $(5 \mu \mathrm{g})$, fosfomicina $(200 \mu \mathrm{g})$, tetraciclina $(30 \mu \mathrm{g})$, sulfametoxazol + Trimetoprim $(23,75+1,25 \mu \mathrm{g})$, nitrofurantoína $(300 \mu \mathrm{g})$.

A inoculação ocorreu por suspensão bacteriana. As placas 
contendo ágar Muller-Hinton e semeadas com a suspensão bacteriana e com discos de antimicrobianos foram incubadas a $37^{\circ} \mathrm{C}$ durante 18 horas. A leitura e interpretação dos diâmetros dos halos foram efetuadas segundo as diretrizes do Clinical and Laboratory Standards Institute (CLSI 2008, CLSI 2011). Foram considerados isolados multirresistentes os que apresentaram resistência a três ou mais classes de antimicrobianos (Magiorakos et al. 2011).

Cálculo estatístico (significância $\mathrm{p}<0,05$ ). Entre isolados de E. coli provenientes de frangos e de tratadores considerados como suscetíveis, resistentes para uma classe, resistentes para duas classes, multirresistentes, com resistência e análise estratificada para três categorias de resistências foi adotado o programa Epi Info 6.04 .

Tipagem molecular. Vinte e quatro isolados de E. coli de frangos de criação intensiva e oito de tratadores de criação intensiva, escolhidos por sorteio, e pertencentes aos mesmos estabelecimentos produtivos foram subcultivadas em ágar sangue para a obtenção de colônias puras e submetidas a técnica de PFGE.

0 preparo dos blocos foi realizado de acordo com Silva et al. (2011). O DNA bacteriano dos isolados de E. coli foi submetido à enzima de restrição Spel (New England Biolab, Inc., Beverly, Mass, USA - 10U por amostra), por 12 a 18 horas a uma temperatura de $37^{\circ} \mathrm{C}$. A eletroforese foi realizada em gel de agarose a $1 \%$ no sistema CHEF-DR III (Bio-Rad, Richmond, CA, EUA) e os padrões de variação da corrente elétrica (swiTIh time) foram de 5 segundos (inicial) e final de 90 segundos. A eletroforese foi realizada por um período de 24 horas, em solução $0,5 x$ TBE (Tris $0,089 \mathrm{M}$; ácido bórico 0,089 $\mathrm{M}$; EDTA, 0,002 M) à temperatura de $13^{\circ} \mathrm{C}$ e utilizando uma corrente elétrica de 200 volts $(6 \mathrm{~V} / \mathrm{cm})$. Os géis foram corados com brometo de etídio $(0,08 \mu \mathrm{g} / \mathrm{mL})$ por uma hora, descorados em água destilada por mais uma hora e fotografados sob luz ultravioleta (Silva et al. 2011).

Os perfis migratórios obtidos após a fotografia do gel foram analisados visualmente seguindo os critérios de Tenover et al. (1995). Para efeito de representação foram utilizados letras e números, uma letra maiúscula representa um clone; e para cada perfil relacionado a este clone, ou seja, um subtipo é utilizado à mesma letra maiúscula seguida de um número arábico.

A similaridade foi avaliada conforme o protocolo da Pulsenet. Os padrões de macrorestrição com Xbal foram analisados com o software BioNumerics (Applied Maths, A., USA) utilizando método UPGMA ("Unweighted Pair Group Method with Arithmetic Mean") e o coeficiente de Dice interpretados conforme Tenover et al. (1995). Na comparação da similaridade entre clusters aplicou-se $1,5 \%$ na tolerância de corte.

\section{RESULTADOS E DISCUSSÃO}

\section{Resistência por classe antimicrobiana}

Todos os isolados de frangos de criação intensiva foram resistentes à ampicilina, enquanto nos isolados de frangos de criação de subsistência o perfil foi de 20\% (Quadro 1).

A resistência de $E$. coli às cefalosporinas de $3^{\underline{a}} \mathrm{e} 4^{\mathrm{a}}$ geração nos isolados de $E$. coli provenientes de frangos de corte de criação intensiva foi elevada enquanto todos os isolados de $E$. coli de frangos em criação de subsistência foram suscetíveis. A resistência às quinolonas nos isolados de criação intensiva foi de $62 \%$ para o ácido nalidíxico e de $23 \%$ para a enrofloxacina, enquanto nos isolados de subsistência foi de $5 \%$ e $2 \%$, respectivamente. A resistência à tetraciclina e ao trimetoprim-sulfametoxazol foi superior nos isolados de criação intensiva ( $83 \%$ e $45 \%$, respectivamente) do que nas de subsistência (33\% e 8\%, respectivamente). Os isolados de E. coli de frangos em criação de subsistência apresentaram resistência superior à fosfomicina e à nitrofurantoína, antimicrobianos utilizados frequentemente na terapêutica da infecção do trato urinário humano.

Em tratadores de frangos de criação intensiva, o percentual de resistência para a ampicilina foi superior à apresentada pelos isolados de E. coli dos tratadores de frangos de subsistência (60\% de 20\%) (Quadro 2).

A frequência de resistência à tetraciclina e trimetoprim-sulfametoxazol foi semelhante nos isolados de tratadores de criação intensiva e nos de subsistência. A resistência às cefalosporinas de $3^{a}$ geração, quinolonas, fosfomicina e nitrofurantoína nos isolados dos tratadores foi baixa nos dois sistemas. Porém, a resistência de $25 \%$ para a ciprofloxacina nos tratadores de criação intensiva em relação aos 5\% dos tratadores de subsistência merece ser considerada. Para o cefepime (cefalosporina de $4 \underline{a}$ geração) não foram verificadas resistências nos isolados de E. coli dos tratadores.

$\mathrm{Na}$ análise da resistência por classe de antimicrobiano em E. coli de frangos de criação intensiva estas apresentaram $60(100 \%)$ resistência para $\beta$-lactâmicos, 40 (66\%)

Quadro 1. Perfil de resistência de isolados de Escherichia coli de frangos de criação intensiva e de frangos de Criação de subsistência

\begin{tabular}{|c|c|c|c|c|c|c|}
\hline \multirow[t]{2}{*}{ Antimicrobianos } & \multicolumn{3}{|c|}{$\mathrm{CI}$} & \multicolumn{3}{|c|}{$\mathrm{CS}$} \\
\hline & $\mathrm{R} \%$ & I $\%$ & S \% & $\mathrm{R} \%$ & I $\%$ & $\mathrm{~S} \%$ \\
\hline Ampicilina $(10 \mu g)$ & 100 & 0 & 0 & 20 & 0 & 80 \\
\hline Ceftriaxona $(30 \mu \mathrm{g})$ & 48 & 0 & 52 & 0 & 0 & 100 \\
\hline Ceftazidima $(30 \mu \mathrm{g})$ & 5 & 5 & 90 & 0 & 0 & 100 \\
\hline 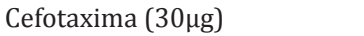 & 43 & 2 & 55 & 0 & 2 & 98 \\
\hline Cefepime $(30 \mu \mathrm{g})$ & 20 & 15 & 65 & 0 & 0 & 100 \\
\hline Ácido Nalidíxico $(10 \mu \mathrm{g})$ & 62 & 8 & 30 & 5 & 0 & 95 \\
\hline Enrofloxacina $(5 \mu \mathrm{g})$ & 23 & 40 & 37 & 2 & 7 & 91 \\
\hline 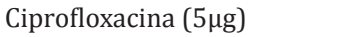 & 23 & 10 & 77 & 4 & 2 & 94 \\
\hline Fosfomicina $(200 \mu \mathrm{g})$ & 4 & 0 & 96 & 16 & 0 & 84 \\
\hline Tetraciclina $(30 \mu \mathrm{g})$ & 83 & 2 & 15 & 33 & 0 & 67 \\
\hline $\begin{array}{l}\text { Sulfametoxazol+Trimetoprim } \\
(23,75+1,25 \mu \mathrm{g})\end{array}$ & 45 & 2 & 53 & 8 & 0 & 92 \\
\hline 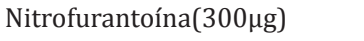 & 10 & 4 & 86 & 16 & 4 & 80 \\
\hline
\end{tabular}

$\overline{\mathrm{CI}}=$ Criação intensiva, $\mathrm{CS}=$ Criação de subsistência, $\mathrm{R}=$ Resistente, $\mathrm{I}=$ Intermediário, $\mathrm{S}=$ Suscetível.

Quadro 2. Perfil de resistência de isolados de Escherichia coli de tratadores de frangos de criação intensiva e de tratadores de frangos de criação de subsistência

\begin{tabular}{|c|c|c|c|c|c|c|}
\hline \multirow[t]{2}{*}{ Antimicrobianos } & \multicolumn{3}{|c|}{ TI } & \multicolumn{3}{|c|}{ TS } \\
\hline & $\mathrm{R} \%$ & I $\%$ & S \% & $\mathrm{R} \%$ & I $\%$ & $\mathrm{~S} \%$ \\
\hline Ampicilina $(10 \mu \mathrm{g})$ & 60 & 0 & 40 & 20 & 0 & 80 \\
\hline Ceftriaxona $(30 \mu \mathrm{g})$ & 5 & 0 & 90 & 0 & 0 & 100 \\
\hline Ceftazidima $(30 \mu \mathrm{g})$ & 0 & 5 & 100 & 0 & 15 & 85 \\
\hline 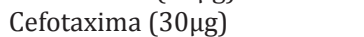 & 15 & 2 & 85 & 15 & 6 & 80 \\
\hline Cefepime $(30 \mu \mathrm{g})$ & 0 & 15 & 100 & 0 & 0 & 100 \\
\hline Ácido Nalidíxico $(10 \mu \mathrm{g})$ & 25 & 20 & 55 & 20 & 0 & 80 \\
\hline Enrofloxacina $(5 \mu \mathrm{g})$ & 15 & 15 & 70 & 10 & 5 & 85 \\
\hline Ciprofloxacina $(5 \mu \mathrm{g})$ & 25 & 0 & 75 & 5 & 0 & 95 \\
\hline Fosfomicina $(200 \mu \mathrm{g})$ & 0 & 0 & 100 & 5 & 0 & 95 \\
\hline Tetraciclina $(30 \mu \mathrm{g})$ & 45 & 0 & 55 & 30 & 0 & 70 \\
\hline $\begin{array}{l}\text { Sulfametoxazol+Trimetoprim } \\
(23,75+1,25 \mu \mathrm{g})\end{array}$ & 30 & 0 & 70 & 35 & 0 & 65 \\
\hline 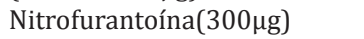 & 5 & 4 & 95 & 10 & 10 & 80 \\
\hline
\end{tabular}

TI = Criação intensiva, TS = Criação de subsistência, $\mathrm{R}=$ Resistente, $\mathrm{I}=$ Intermediário, $\mathrm{S}=$ Suscetível. 
para quinolonas, $50(83 \%)$ para tetraciclina, $27(45 \%)$ para sulfonamidas. Os isolados de E. coli de tratadores de frangos de corte de criação intensiva apresentaram 12 (60\%) para $\beta$-lactâmicos e oito (40\%) para quinolonas. As resistências nos isolados de frangos de criação de subsistência foram maiores do que isolados de frangos de criação de intensiva apenas para fosfomicina e nitrofuranos. Apenas em sulfonamidas que os isolados de tratadores de subsistência excederam aos isolados de tratadores de criação intensiva e de isolados de frangos de criação de subsistência (Fig.1).

\section{Perfil de resistência e multirresistência}

Elevado número de isolados de E. coli de frangos em criação de subsistência (28 de 60, 46.6\%) apresentaram-se suscetíveis a todos os antimicrobianos testados em contraste com os isolados de criação intensiva que foram principalmente multirresistentes (49 de 60, 81.6\%).

Os isolados de E. coli de tratadores de criação de subsistência e intensiva apresentaram perfil concordante de 8/20 (40\%) e $7 / 20$ (35\%) para suscetibilidade a multirresistência a todos os antimicrobianos testados, respectivamente.

A análise estatística (significância $\mathrm{p}<0,05$ ) entre as amostras de E. coli de frangos e de tratadores para suscetível, resistente para uma classe, resistentes para duas

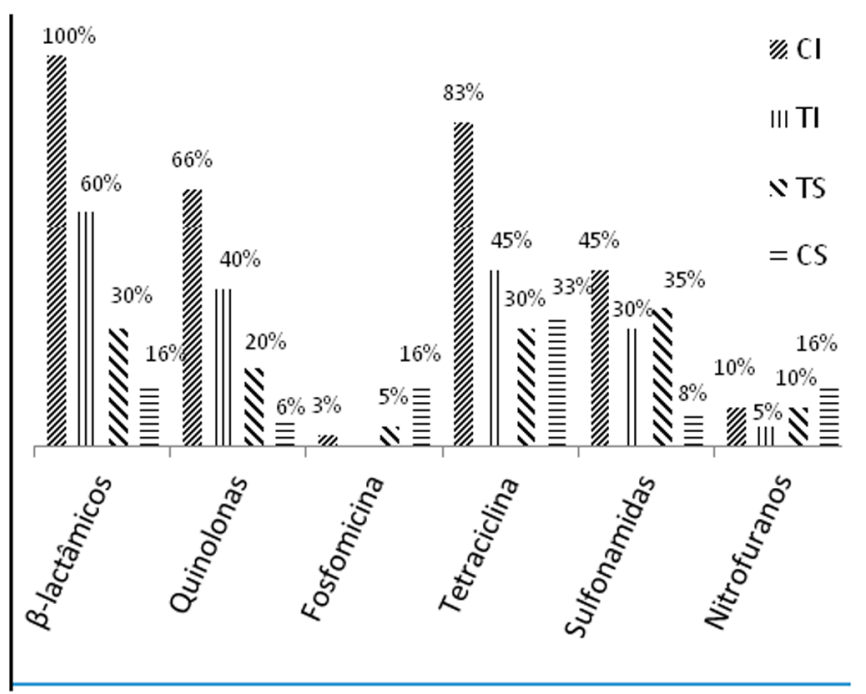

Fig.1. Percentagem de isolados resistentes para cada classe de antimicrobiano; $\mathrm{CI}=$ Criação intensiva, $\mathrm{TI}=$ Tratadores de criação intensiva, TS = Tratadores de criação de subsistência, CS = Criação de subsistência.

Quadro 3. Análise estatística

\begin{tabular}{lcccc}
\hline & CI X TI & CI X CS & TI X TS & CS X TS \\
\hline Suscetível & NS & NS & NS & NS \\
Res. para 1 classe & NS & S & NS & NS \\
Res. para 2 classes & NS & S & NS & NS \\
Multirresistentes & S & S & NS & NS \\
Com resistência & S & S & NS & NS \\
A.E.C.R. & S & S & S & NS
\end{tabular}

$\overline{\mathrm{CI}}=$ Criação intensiva, $\mathrm{TI}=$ Tratadores de criação intensiva, $\mathrm{CS}=$ Criação de subsistência, $\mathrm{TS}=$ Tratadores de subsistência, $\mathrm{S}=$ Significativo $(\mathrm{p}<0,05), \mathrm{NS}=$ Não significativo, A.E.C.R. = Análise estratificada para três categorias de resistência. classes, multirresistentes, com resistência e análise estratificada para três categorias de resistências apontou os resultados que constam no Quadro 3.

\section{Resistências simultâneas às cefalosporinas de $3^{\underline{a}}$ e $4^{\underline{a}}$ geração e às fluorquinolonas}

Doze (19,35\%) isolados de E. coli de frangos de criação intensiva apresentaram frequência de resistência simultânea às cefalosporinas de $3^{\text {a }}$ geração e às fluorquinolonas, enquanto em frangos de criação de subsistência e em tratadores não ocorreu esta co-resistência.

Este estudo demonstrou elevada resistência para a ampicilina (100\%), cefalosporinas de $3^{\mathrm{a}}$ e $4^{\mathrm{a}}$ geração, quinolonas, tetraciclina e ao sulfametoxazol-trimetoprim nos isolados de E. coli de frangos de criação intensiva comparativamente aos isolados de E. coli de frangos de criação de subsistência, o que pode indiciar a pressão seletiva exercida pelo uso de antimicrobianos na produção intensiva avícola. No Brasil, não existem dados sobre as classes e volume de antimicrobianos consumidos na produção animal. No entanto, na Europa, em 2011, as vendas totalizaram 55.872 toneladas de antimicrobianos para animais de produção e corresponderam a $37 \%$ de tetraciclinas, $23 \%$ de penicilinas, $11 \%$ de sulfonamidas e $7 \%$ de polimixinas (Esvac 2013).

Em relação aos estudos com isolados patogênicos de $E$. coli causadores de colisepticemia e celulite aviária, a resistência à ampicilina encontrada por Ozawa (2008) no Japão, Yang et al. (2004) na China, Zakeri e Kashefi (2012) no Irã foi de $77 \%, 79 \%, 58 \%$, respectivamente. Para as cefalosporinas de 3a geração, Abreu et al (2010) reportaram 65\% de resistência para a ceftazidima em isolados de E. coli patogênica de codornas sob inspeção sanitária. Nos demais estudos, estes antimicrobianos não compuseram o padrão dos testes de suscetibilidade utilizado, talvez por se tratarem de antimicrobianos de prescrição humana.

Para a enrofloxacina, Zanatta (2004) em São Paulo, constatou $62 \%$ de resistência em colibacilose, enquanto Ozawa (2008), no Japão, encontrou 21\% e Yuan et al. (2009) na China, $80 \%$. As elevadas resistências à tetraciclina nos isolados de E. coli dos frangos de criação intensiva (83\%) refletem os efeitos dos usos frequentes de antimicrobiano na criação intensiva. Resultados semelhantes de resistência à tetraciclina no Brasil foram verificados por Zanatta (2004) que (76\%) e Aquino, em Londrina (63\%). Zakeri e Kashefi (2012), no Irã, constataram 99\% em colibacilose. A falta de informações sobre volume e princípios ativos de antimicrobianos mais consumidos no Brasil, tanto no setor da produção animal, quanto da saúde humana, dificulta uma leitura mais acurada do contexto da seleção de resistências bacterianas.

Quanto aos outros estudos para o sulfametaxozol + trimetoprim em isolados de E. coli de aves com quadro de colibacilose, as resistências constatadas por Alcântara (2011) foram de 90\%, enquanto Ozawa (2008), no Japão, encontrou $21 \%$ e Yuan et al. (2009) na China, constataram 90\%.

Neste estudo, as altas resistências para fosfomicina e nitrofurantoína em isolados de $E$. coli de frangos de criação de subsistência, antimicrobianos de utilização exclusivamente 
humana para tratamento das infecções do trato urinário, poderiam ter ocorrido pelo contato das aves com resíduos de antimicrobianos de prescrição humana descartados no lixo doméstico e ambiente aberto de acesso às aves (Regitano \& Leal 2010). Esta prática de descarte é comum em comunidades do interior.

A resistência à ampicilina de $60 \%(12 / 20)$ em isolados de E. coli de tratadores de criação intensiva foi superior em relação aos tratadores de criação de subsistência, e em isolados de E. coli $(44,1 \%)$ de pacientes ambulatoriais em Curitiba (Rocha, Tuon e Johnson 2012). Estas resistências em tratadores podem ter ocorrido pelo contato direto com as aves ou na manipulação dos antimicrobianos.

Para as resistências à nitrofurantoína, em $5 \%(1 / 20)$ dos tratadores de criação intensiva e 10\% em tratadores de criação de subsistência estiveram na média constatada entre pacientes ambulatoriais. Rocha, Tuon e Johnson (2012) em Curitiba diagnosticam 4\%; Correia et al. (2007), em Portugal, constataram 1\%; e Esparis et al. (2006), no Rio de Janeiro, $0 \%$ em pacientes ambulatoriais com infecções do trato urinário causadas por E. coli.

As resistências em $30 \%(6 / 20)$ para sulfametaxozol-trimetoprim em isolados de $E$. coli de tratadores de frangos de criação intensiva e 35\% (7/20) para isolados de E. coli de tratadores de subsistência, aproximaram-se aos $42 \%$ de Esparis et al. (2006), no Rio de Janeiro e aos 34,2 \% encontrados por Rocha, Tuon \& Johnson (2012) em Curitiba. Todos os percentuais resultam de estudos com pacientes ambulatoriais.

Para ciprofloxacina as resistências de $25 \%(5 / 20)$ em isolados de E. coli em tratadores de frangos de corte criação intensiva e 5\% (1/20) em isolados de E. coli de tratadores de frangos de criação de subsistência refletem a existência de pressão seletiva para este antimicrobiano. Este fenômeno pode ocorrer por resistência cruzada. Em Curitiba Rocha, Tuon \& Johnson (2012) encontraram 17,2\% de resistência à ciprofloxacina e Levofloxacina. Braios (2009) constatou $14 \%$ de resistência à ciprofloxacina em cidade do interior paulista. Os resultados apontaram resistência superior nos isolados de E. coli de tratadores de frangos de corte de criação intensiva em relação aos demais estudos sobre resistências de E. coli em pacientes ambulatoriais.

Não foram encontrados resultados para a enrofloxacina nos demais estudos em humanos. Chen et al. (2011) na China pesquisaram os efeitos destes antimicrobianos na microbiota fecal humana e concluíram que ela afeta a qualidade, a diversidade de bactérias e aumenta a resistência de toda a microbiota intestinal à ciprofloxacina.

Porém, a discreta diferença entre os $15 \%(3 / 20)$ de resistência para enrofloxacina verificados em isolados de $E$. coli em tratadores de frangos de corte criação intensiva, em relação aos 10\% (2/20) em isolados de E. coli de tratadores de frangos de criação de subsistência, refletem a existência de pressão seletiva em ambos, cuja origem pode ter ocorrido por resistência cruzada em função do uso de medicamentos na saúde humana. É importante destacar que todos os tratadores de subsistência são do sexo feminino e o uso de antimicrobianos no tratamento de infecções urinárias pode ter intensificado a seleção destas resistências.

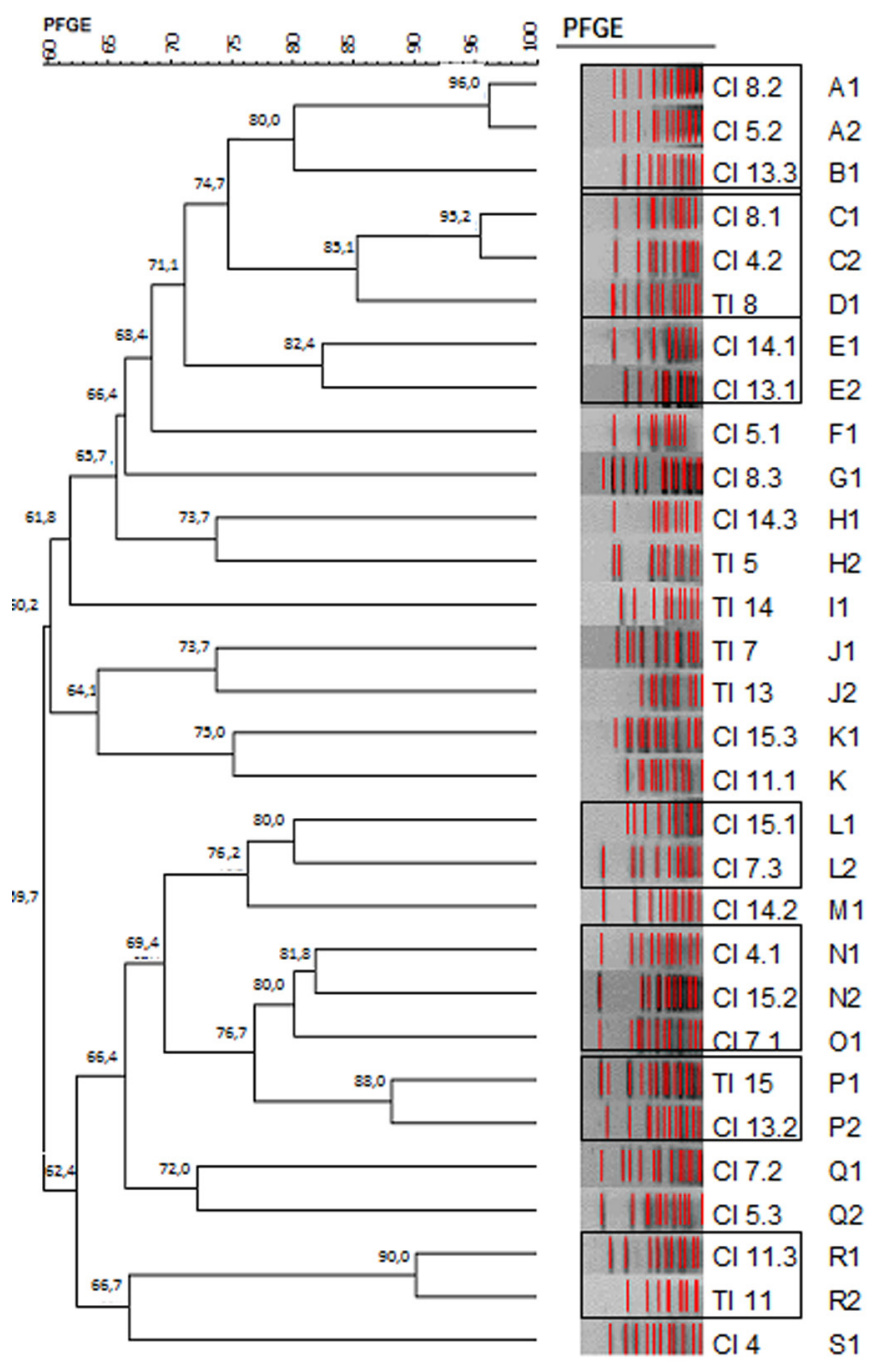

Fig.2. Dendograma do PFGE de isolados de Escherichia coli de frangos de corte de criação itensiva e de tratadores de frangos de criação intensiva.

\section{Perfil de similaridade genotípica dos isolados (PFGE)}

Dois isolados foram classificados como não tipáveis por PFGE. Sete clusters ( "A", “B” “C”, “E”, "L", "N", “O”) de isolados de E. coli de frangos apresentaram similaridade genotípica acima de $80 \%$. Os clusters " $\mathrm{A}$ " e "C" apresentaram similaridade superior a $95 \%$ (Fig.2).

$\mathrm{Na}$ análise entre isolados de frangos e de tratadores, três clusters apresentaram similaridade superior a $80 \%$. Dois destes clusters foram de isolados bacterianos de frangos e de tratadores colhidos no mesmo estabelecimento produtivo, a citar, o isolado CI 8.1 do cluster " $\mathrm{C}$ " com o isolado TI 8 do cluster "D" e os isolados do cluster "R". No cluster " $\mathrm{R}$ " os isolados apresentaram similaridade superior a $90 \%$. Porém os isolados CI 4.2 do cluster "C" e a amostra TI 8 do cluster " $\mathrm{D}$ ", apresentaram similaridade de $85 \%$, não foram colhidas no mesmo aviário, assim como os isolados do cluster " $\mathrm{P}$ " que apresentaram similaridade de $88 \%$.

Foi encontrada similaridade superior a $95 \%$ entre dois clusters de isolados de E. coli de frangos de dois aviários diferentes e localizados entre si a distâncias superiores a $2 \mathrm{~km}$. No entanto, estes isolados apresentaram diferentes 
perfis de resistência e a diferentes classes de antimicrobianos.

Os resultados do PFGE apontam para o potencial de disseminação de bactérias no ambiente e por consequência das resistências. Esta forma de disseminação entre isolados de E. coli de frangos, e destes com isolados de tratadores, também foi verificada por Van Den Bogaard et al. (2001) em estudo na Holanda ao comparar isolados de $E$. coli de frangos e isolados de E. coli de tratadores. Para explicar o fenômeno, os autores argumentaram pela colonização do trato digestório dos tratadores por bactérias comensais de frangos em decorrência do contato direto com as aves.

$\mathrm{O}$ fato de não ter sido encontrada clonalidade entre isolados de E. coli de tratadores e isolados de E. coli de frangos de criação intensiva pode ter ocorrido devido ao número de colônias selecionadas, embora o número de isolados do estudo tivesse significância estatística. Porém, a existência de similaridade genotípica acima de $80 \%$ em três clusters entre estes isolados, e ter sido encontrada clonalidade entre isolados de frangos, não descarta a possibilidade de terem existidos clones de $E$. coli entre isolados de $E$. coli de frangos de corte de criação intensiva e de isolados de E.coli de tratadores de criação intensiva na região de colheita das amostras.

\section{CONCLUSÕES}

A alta resistência aos antimicrobianos $\beta$-lactâmicos em E. coli comensal de frangos de corte de criação intensiva e nos tratadores destas aves, em comparação com o perfil de resistência em isolados de E. coli de frangos de criação de subsistência e dos isolados de E. coli de tratadores de frangos de subsistência, é um indicativo da existência de pressão seletiva na microbiota nas aves e nos tratadores em decorrência do uso de antimicrobianos.

É relevante a similaridade genotípica, acima de $80 \%$, encontrada pela técnica de PFGE entre três isolados de $E$. coli de frangos de corte de criação intensiva e três isolados de $E$. coli de tradores, a considerar que dois destes isolados de $E$. coli de frangos e dois de tratadores foram colhidos em diferentes aviários.

A distância entre os aviários, não superior a $2 \mathrm{~km}$, pode ter contribuido nestes resultados.

A disseminação de bactérias, como E. coli, pode ocorrer por meio de animais domésticos e de vida livre, partículas de dejetos dos frangos transportados pelo vento, pelas vestimentas da equipe técnica da agroindústria durante visitação, pelos caminhões que transportam as rações e pelas equipes de trabalho das agroindústrias durante o carregamento dos frangos para o abate.

\section{REFERÊNCIAS}

Abreu D.L.C., Franco R.M., Nascimento E.R., Pereira V.L.A., Alves F.M.X. \& Almeida J.F. 2010. Perfil de sensibilidade antimicrobiana e detecção do gene ISS pela reação em cadeia da polimerase na tipificação de Escherichia coli patogênica em codornas de corte sob inspeção sanitária. Pesq. Vet. Bras. 30(5):406-410.

Alcântara A.C.M. 010. Realização de antibiograma e detecção de genes de resistência pela reação em cadeia da polimerase (PCR) em cepas de $E$. coli isoladas de celulite aviária coletadas de abatedouros frigoríficos do
Distrito Federal, Brasília. Tese de Doutorado. Disponível em <http:// bdm.bce.unb.br/handle/10483/1809> Acesso em 18 abr. 2012.

Bauer A.W., Kirby W.M., Sherris J.C. \& Turck M. 1966. Antibiotic susceptbility testing by a standardized single disk method. Am. J. Clin. Pathol. 45:493-496.

AGISAR 2012. Critically Important Antimicrobials for Human Medicine. 3rd ed. Disponível em <http://apps.who.int/iris/bitstream/10665/77376/ 1/9789241504485_eng.pdf> Acesso em 6 dez. 2013.

Blanco J.E., Blanco M., Mora A. \& Blanco J. 1997. Prevalence of bacterial resistance to quinolones and other antimicrobials among avian Escherichia coli strains isolated from septicemic and healthy chickens in Spain. J. Clin. Microbiol. 35(8):2184-2185.

Braios A., Turatti T.F., Meredija L.C.S., Campos T.R.S. \& Denadai F.H.M. 2009. Infecções do trato urinário em pacientes não hospitalizados: etiologia e padrão de resistência aos antimicrobianos. Bras. Patol. Med. Lab. 45(6):449-456.

Chen T., Yuan J,. Feng X., Wei H. \& Hua W. 2011. Effects of enrofloxacin on the human intestinal microbiota in vitro. Int. J. Antimicrobial Agents 22(5):567-571.

CLSI 2008. Performance Standards for Antimicrobial Disk and Diluition Susceptibility Tests for Bacteria Isolated from Animals: approved standard. 3rd ed. Clinical and Laboratory Standards Institute, 940 West Road, Suite 1400, Wayne, Pennsylvania 19087-1898, USA, 28(8):2-11.

CLSI 2011. Performance Standarsds for Antimicrobial Suceptibily Testing Twenty: first information suplement. Clinical and Laboratory Standards Institute, 940 West Road, Suite 1400, Wayne, Pennsylvania, 31(1).

Correia C., Costa E., Peres A., Alves M., Pombo G. \& Estevinho L. 2007. Etiologia das infecções do tracto urinário e sua susceptibilidade aos antimicrobianos. Acta Med. Port. 20:543-549.

Dheilly A., Bouder A., Le Devendec L., Hellard G. \& Kempf I. 2011. Clinical and microbial efficacy of antimicrobial treatments of experimental avian colibacillosis. Vet. Micorbiol. 149(3/4):422-429.

Dufour-Zavala L. 2008. Laboratory Manual for the Isolation, Identification, and Characterization of Avian Pathogens. 5th ed. American Association of Avian Pathologists, Georgia.

Dziva F. \& Stevens S.M.P. 2008. Colibacillosis in poultry: unravelling the molecular basis of virulence of avian pathogenic Escherichia coli in their natural hosts. Avian Pathol. 37(4):355-366.

Esparis C.M., Teixeira L.M., Irino K., Gil P.F., Almeida M.M.T.B., Lopes G.S., Bravo V.L.R., Pacheco R.S. \& Regua-Mangia A.H. 2006. Aspectos biológicos e moleculares de amostras uropatogênicas de Escherichia coli isoladas na cidade do Rio de Janeiro. Revta Soc. Bras. Med. Trop. 39(6):573576.

Esvac 2013. Sales of veterinary antimicrobial agents in 25 EU/EEA countries in 2011. Disponível em <http://www.ema.europa.eu/docs/en_GB/ document_library/Report/2013/10/WC500152311.pdf> Acesso em 10 dez. 2013.

Ewers C., Bethe A., Semmler T., Guenter S. \& Wieler L.H. 2012. Extendedspectrum lactamase-producing and $A m p C$-producing Escherichia coli from livestock and companion animals, and their putative impact on public helth: a global perspective. Clin. Microbiol. Infect. 18(7):646-655.

Gould D. 2010. Causes, prevention and treatment of Escherichia coli infection. Nursing Standard 24(31):50-56.

Johnson J.R., Sannes M.R., Croy C., Johnston B., Clabots C., Kuskowski M.A., Bender J., Smith K.E., Winokur P.L. \& Belongia E.A. 2007. Antimicrobial drug-resistant Escherichia coli from humans and poultry products, Minnesota and Wisconsin, 2002-2004. Emerg. Infect. Dis. 13(6):838846.

Knöbl T., Moreno A.M., Paixão R., Gomes T.A., Vieira M.A., Silva L.D., Blanco J.E. \& Ferreira A.J. 2012. Prevalence of avian pathogenic Escherichia coli clone harboring sfa gene in Brazil. Scient. World J. 2012:1-7.

Magiorakos A.P., Srinivasan A., Carey R.B., Carmeli Y., Falagas M.E., Giske C.G., Harbarth S., Hindler J.F., Kahlmeter G., Olsson-liljequist B., Paterson D.L., Rice L.B., Stelling J., Struelens M.J., Vatopoulos A. \& Weber J.T. 2011. Multidrug-resistant, extensively drug-resistant and pandrug-resistant bacteria: an international expert proposal for interim standard 
definitions for acquired resistance. Eur. Soc. Clin. Microbiol. Infect. Dis. 18(3):268-281.

Manges A.R. \& Johnson J.R. 2012. Food-borne origins of Escherichia coli causing extraintestinal infections. Clin. Infect. Dis. 55(5):712-719.

Ozawa M., Harada K., Kojima A., Asai T. \& Sameshima T. 2008. Antimicrobial susceptibilities, serogroups, and molecular haracterization of avian pathogenic Escherichia coli isolates in Japan. Avian Dis. 52(3):391-398.

Regitano J.B. \& Leal R.M.P. 2010. Comportamento e impacto ambiental de antibióticos usados na produção animal brasileira. Revta Bras. Ciênc. Solo 34:601-616.

Rocha J.L., Tuon F.F. \& Johnson J.R. 2012. Sex, drugs, bugs, and age: rational selection of impirical therapy for outpatient urinary tract infection in an era of extensive Antimicrobial resistance. Braz. J. Infect. Dis. 16(2):115121.

Shah P.M. 1991. Ciprofloxacin. Int. J. Antimicrob. Agents 1(2/3):75-96.

Sheerin N.S. 2011. Urinary tract infection. Medicine 39(7):384-389.

Silva F.M., Carmo M.S., Silbert S. \& Gales A.C. 2011. SPM-1-producing Pseudomonas aeruginosa: analysis of the ancestor relationship using multilocus sequence typing, pulsed-field gel electrophoresis, and automated ribotyping. Microb. Drug Resist. 17(2):215-220.

Tenover F.C., Arbeit R.D., Goering R.V., Mickelsen P.A., Murray B.E., Persing D.H. \& Swaminathan B. 1995. Interpreting chromosomal DNA restric- tion patterns produced by Pulsed-Field Gel Electrophoresis: criteria for bacterial strain typing. J. Clin. Microbiol. 33(9):2233-2239.

WHO 2013. Anti-microbial resistance. Disponível em <http://www.who. int/trade/glossary/story004/en/> Acesso em 13 abr. 2013.

Van den Bogaard A.E., London N., Driessen C. \& Stobberingh E.E. 2001. Antibiotic resistance of faecal Escherichia coli poultry, poultry farmers and poultry slaughterers. J. Antimicrob. Chemotherapy 47(6):763-771.

Yang H., Chen S., White D., Zhao S., McDermott P., Walker R. \& Meng J. 2004. Characterization of Multiple-Antimicrobial-Resistant Escherichia Coli Isolates from Diseased Chickens and Swine in China. J. Clin. Microbiol. 42(8):3483-3489.

Yuan L., Liu J.H., Hu G.Z., Pan Y.S., Liu Z.M., Mo J. \& Wei Y.J. 2009. Molecular characterization of extended-spectrum $\beta$-lactamase-producing Escherichia coli isolates from chickens in Henan Province, China. J. Med. Microbiol. 58(11):1449-1453.

Zakeri A. \& Kashefi P. 2012. Antimicrobial susceptibilities of avian Escherichia coli isolates in Tabriz, Iran. African J. Biotechnol. 11(19):44674470.

Zanatta G.F., Kanashiro A.M.I., Castro A.G.M., Cardoso A.L.S.P., Tessari E.N.C. \& Pulici S.C.P. 2004. Sucetibilidade de amostras de Escherichia coli de origem aviária a antimicrobianos. Arqs Inst. Biológico, São Paulo, 71(3):283-286. 